\title{
Pericardial Effusion Presenting with Anuric Acute Renal Failure and Hepatocellular Damage
}

\author{
Raza Khan, MD ${ }^{1}$ \\ Charles Gessert, MD, MPH${ }^{2}$ \\ Stephen Bockhold, MD ${ }^{3}$ \\ ${ }^{1}$ Department of Hospital Medicine, SMDC Health \\ System, Duluth, Minnesota \\ ${ }^{2}$ Department of Education and Research, SMDC \\ Health System, Duluth, Minnesota \\ ${ }^{3}$ Duluth Family Practice Residency Program, \\ Duluth, Minnesota
}

\begin{abstract}
A 50-year-old male with anuria, creatinine of 5.5 and potassium of 6.5 was referred to our hospital for hemodialysis. Before hemodialysis could be initiated, his blood pressure dropped and liver function tests were found to be increasing rapidly. This prompted us to look for cardiac causes of liver ischemia. An echocardiogram was non-diagnostic due to the patient's obese body habitus. Pericardial fluid was documented on CT scan. Pericardiocentesis was performed and nearly $1500 \mathrm{ml}$ of bloody pericardial fluid was removed. This resulted in immediate urine output, with $80 \mathrm{ml}$ in the first hour, and an increase in blood pressure. Journal of Hospital Medicine 2009;4:68-70. ( 2009 Society of Hospital Medicine.
\end{abstract}

KEYWORDS: acute renal failure, obesity, pericarditis.

들 days prior to admission to our hospital, a 50-year-old ᄃ morbidly obese patient presented to his primary care clinic, complaining of weakness, dizziness, and symptoms consistent with paroxysmal nocturnal dyspnea. He had a history of diabetes mellitus type 2, hypertension, chronic renal insufficiency with baseline creatinine in the range of $2.0 \mathrm{mg} / \mathrm{dL}$, and atrial fibrillation on warfarin (10 mg/day). He was given diuretics and sent home. Two days later, the patient presented to a rural emergency department, complaining of leg pain and swelling. He was given cephalexin for cellulitis and discharged home. The evening prior to admission to our hospital, the patient developed sharp leftsided chest pain, orthopnea, and worsening dyspnea. He again presented to the rural emergency department and was found to have a blood pressure of 60/40 mm Hg. He was admitted and given boluses of intravenous fluids and a dose of ceftriaxone. Laboratory studies at that time demonstrated the following: sodium, $130 \mathrm{mEq} / \mathrm{L}$; potassium, $6.5 \mathrm{mEq} / \mathrm{L} ; \mathrm{CO}_{2}, 21 \mathrm{mEq} / \mathrm{L}$; blood urea nitrogen (BUN), $105 \mathrm{mg} / \mathrm{dL}$; creatinine, $5.5 \mathrm{mg} / \mathrm{dL}$; alkaline phosphatase, $330 \mathrm{IU} / \mathrm{L}$; aspartate aminotransferase (AST), 507 IU/L; and alanine aminotransferase (ALT), 145 IU/L. The following morning, he was oliguric, and his liver transaminases had increased to an AST level of 1862 IU/L and an ALT level of 1055 IU/L. At this point, he was transferred to our hospital for hemodialysis with a diagnosis of acute oliguric renal failure.

On admission to our hospital, the patient was afebrile, had a blood pressure of $112 / 80 \mathrm{~mm} \mathrm{Hg}$, and was hypoxic with an $\mathrm{O}_{2}$ saturation of $93 \%$ on $4 \mathrm{~L}$ of oxygen by nasal cannula. His weight was $201 \mathrm{~kg}$. He was alert and cooperative. Heart sounds were distant but had a regular rate and rhythm without murmurs, rubs, or gallops. No jugular venous pulsations were appreciated. 
He had decreased lung sounds throughout both lung fields. His abdomen was morbidly obese and nontender. Extremities demonstrated chronic venous stasis changes with multiple superficial ulcers and bilateral pitting edema.

In light of the elevated liver function tests reported by the referring hospital, these studies were repeated on arrival, revealing an AST level of $4780 \mathrm{IU} / \mathrm{L}$ and an ALT level of $1876 \mathrm{IU} / \mathrm{L}$. Other initial laboratory studies demonstrated a BUN level of $116 \mathrm{mg} / \mathrm{dL}$, a creatinine level of $6 \mathrm{mg} / \mathrm{dL}$, a potassium level of $7.2 \mathrm{mEq} / \mathrm{L}$, negative cardiac enzymes, and an international normalized ratio greater than 9.0. A urinalysis could not be performed because the patient was anuric. An electrocardiogram demonstrated a normal sinus rhythm with a right bundle branch block and nonspecific ST segment and T wave changes. There was no prior electrocardiogram available for comparison. Blood acetaminophen level and infectious hepatitis panels were negative.

Within 1 hour of admission, the patient became hypotensive, with his systolic blood pressure decreasing into the 70s. Over the next 24 hours, the patient was treated with intravenous fluids and pressors (neosynephrine) but remained hypotensive, with his systolic blood pressure in the range of 64 to $80 \mathrm{~mm} \mathrm{Hg}$.

At 14 hours after admission, liver function tests were repeated and revealed further elevation of his transaminases (AST, 5135 IU/L; ALT, 2468 IU/L). The diagnosis of shock liver was entertained. A portable chest X-ray (CXR) demonstrated an enlarged cardiac silhouette suggestive of cardiomegaly or pericardial effusion. No prior CXR was available for comparison. An echocardiogram showed probable effusion but was not diagnostic secondary to his body habitus and poor windows. A computed tomography (CT) scan confirmed a large pericardial effusion. This finding, in combination with diminished heart sounds and hypotension, presented a clinical picture consistent with cardiac tamponade. The patient underwent a subxiphoid pericardial window, and $1.5 \mathrm{~L}$ of bloody effusion was removed. Intraoperatively, his systolic blood pressure immediately improved from 95 to $135 \mathrm{~mm} \mathrm{Hg}$. Urine output increased from 0 to $80 \mathrm{~mL} /$ hour in the first hour. Five days later, his creatinine returned to his baseline of $1.9 \mathrm{mg} / \mathrm{dL}$, and other laboratory values had decreased: AST, $306 \mathrm{IU} / \mathrm{L}$; ALT, $520 \mathrm{IU} / \mathrm{L}$; alkaline phosphatase, $122 \mathrm{IU} / \mathrm{L}$; and BUN, $86 \mathrm{mg} / \mathrm{dL}$.

\section{DISCUSSION}

Acute renal failure (ARF) is a common condition, with an incidence of $1 \%$ on admission to the hospital; $70 \%$ of these cases are due to prerenal causes. ${ }^{1}$ Among patients already in the hospital, prerenal azotemia is responsible for $21 \%$ to $39 \%$ of cases of $\mathrm{ARF}^{2,3}$ Prerenal ARF is most commonly caused by hypotension, which may be cardiogenic, hypovolemic, septic, or due to vasodilatation. Adrenal insufficiency and other etiologies should also be considered. In our patient, chronic renal failure and superimposed hypotension contributed to acute oliguric renal failure. In searching for the etiology of his hypotension, we concentrated on cardiogenic causes in view of his enlarged cardiac silhouette.

In considering the cardiogenic causes of hypotension, we did not initially focus on tamponade. Although oliguria with renal failure is recognized as a complication of cardiac tamponade, relatively little has been written about ARF as the presenting problem in tamponade. The literature is limited to a few case reports, such as those described by Queffeulou et al. $^{4}$ and Saklayen et al. $^{5}$ In our patient, the diagnosis of tamponade was made more difficult by the patient's large size, which made the interpretation of physical findings such as heart sounds more difficult and compromised the quality of essential imaging studies.

His rising transaminases suggested shock liver and eventually provided the key to the patient's diagnosis. In light of his worsening liver function tests, chest pain, shortness of breath, and cardiomegaly on CXR, we focused on a cardiac etiology. An echocardiogram, which often can be used to identify pericardial effusion and hence tamponade, was nondiagnostic because of the patient's obesity. Despite the concerns regarding the weight limits of the examination table, the diagnosis was finally established with a CT scan.

In our patient, no definite cause for his bloody pericardial effusion was found. Cardiac enzymes were negative, and this helped rule out ischemia. Nothing indicative of a neoplasm was seen on the CT of his chest. Blood cultures were negative. Viral studies were not performed, and the effusion was not sent for special studies. He may have had an underlying pericarditis secondary to his chronic kidney disease or a viral syndrome that, combined with his anticoagulation treatment, resulted in a bloody effusion. 
In addition to illustrating the difficulties in properly diagnosing acutely ill morbidly obese patients, this report also demonstrates that in patients with shock liver and ARF, pericardial tamponade may be the culprit. If preliminary studies including CXR and echocardiogram are equivocal and sufficient suspicion remains, then a CT is warranted.

Address for correspondence and reprint requests: Charles Gessert, MD, MPH, Department of Education and Research, SMDC Health System, 400 East 3rd Street, Duluth, MN 55805-1983; Telephone: 218-786-8176; Fax: 218-7278159; E-mail: cgessert@smdc.org or cgessert@yahoo.com

Received 23 July 2007; revision received 20 September 2007; accepted 19 February 2008.

\section{REFERENCES}

1. Kaufman J, Dhakal M, Patel B, Hamburger R. Communityacquired acute renal failure. Am J Kidney Dis. 1991;17: 191-198.

2. Liano F, Pascual J. Epidemiology of acute renal failure: a prospective, multicenter, community-based study. Madrid Acute Renal Failure Study Group. Kidney Int. 1996; 50(3):811-818.

3. Nash K, Hafeez A, Hou S. Hospital-acquired renal insufficiency. Am J Kidney Dis. 2002;39:930-936.

4. Queffeulou G, Vrtovsnik F, Mignon F. Acute renal and hepatic failure: do not miss pericardial tamponade! Nephrol Dial Transplant. 1999;14(9):2260.

5. Saklayen M, Anne VV, Lapuz M. Pericardial effusion leading to acute renal failure: two case reports and discussion of pathophysiology. Am J Kidney Dis. 2002;40(4):837- 841. 